\title{
DECAIMIENTO EXPONENCIAL UNIFORME EN LA VARIABLE TEMPORAL PARA EL ESPACIO DE SEMI-DISCRETIZACIÓN ESPACIAL DE UNA ECUACIÓN DE ONDA CON AMORTIGUAMIENTO Claudio Balcazar Huapaya ${ }^{*}$, Maruja Gavilán Gonzales ${ }^{* *}$, Cristian Loli Prudencio ${ }^{* * *}$, Emilio Castillo Jimenez ${ }^{* * * *}$, Andrés Guardia Cayo ${ }^{* * * * *}$ \& Lucio Malasquez Ruiz ${ }^{* * * * * *}$
}

Resumen.- Nuestro propósito es analizar y alcanzar resultados en esquemas de aproximación numérica clásica de la ecuación de onda unidimensional amortiguada, con relación a la propiedad de decaimiento exponencial de soluciones y determinar si es uniforme con respecto al tamaño de paso de la correspondiente semidiscretización. Consideramos el espacio de semidiscretización en diferencias finitas de una ecuación de onda localmente amortiguado. La razón de decaimiento del sistema semidiscreto resulta depender del tamaño de paso $h$ de la discretización y tiende a cero cuando $h$ va a cero. Probaremos que adicionando un adecuado término de viscosidad numérica, se puede lograr un decaimiento exponencial uniforme de la energía de las soluciones.

Palabras clave: Espacio de semidiscretización en diferencias finitas. Decaimiento exponencial uniforme de soluciones.

\section{UNIFORM EXPONENTIAL DECAY IN THE TEMPORARY VARIABLE SEMI-DISCRETIZATION SPACE OF A DAMPED WAVE EQUATION}

\begin{abstract}
Our purpose is to analyze and to attain result in the classical numerical approximation schemes of the damped wave equation one-dimentional, with relation to exponential decay property of solutions and whether it is uniform with respect to the mesh size. We consider the finite-difference space semi-discretization of a locally damped wave equation. The decay rate of the semi-discrete systems turns out depend on the mesh size $h$ goes to zero. We prove that adding a suitable vanishing numerical viscosity term leads to a uniform exponential decay of the energy of solutions.
\end{abstract}

Key words: Finite-difference space semidiscretizacion. Uniform exponential decay of solutions.

\section{Introducción:}

Consideremos la ecuación de onda unidimensional con amortiguamiento

$$
\left\{\begin{array}{l}
y^{\prime \prime}-y_{x x}+a(x) y^{\prime}=0 \text { en }(0,1) \times(0, \infty) \\
y(0, t)=y(1, t)=0 \text { en }(0, \infty) \\
y(x, 0)=y^{0}(x) \text { en }(0,1) \\
y^{\prime}(x, 0)=y^{1} \text { cn }(0,1)
\end{array}\right.
$$

donde

$$
\begin{aligned}
& y^{0}(x)=\left\{\begin{array}{cc}
x \quad, \quad 0<x \leq 1 / 2 \\
1-x, & 1 / 2 \leq x \leq 1
\end{array}\right. \\
& y^{1}(x)=x^{-1 / 4}, 0<x<1,
\end{aligned}
$$

\footnotetext{
*UNMSM, Facultad de Ciencias Matemáticas. e-mail: cbalcazarh@ummsm.edu.pe

${ }^{* *}$ UNMSM, Facultad de Ciencias Matemáticas, e-mail: mgavilan@unmsm.edu.pe

***UNMSM, Facultad de Ciencias Matemáticas, e-mail: clolip@ummsm.edu.pe

${ }^{* * * *}$ UNMSM, Facultad de Ciencias Matemáticas, e-mail: ecastilloj@ìunmsm.edu.pe

****** UNMSM, Facultad de Ciencias Matemáticas, e-mail: agcdibayo@yahoo.es

******* UNMSM, Facultad de Ciencias Matemáticas, e-mail: luciomalasquez@hotmail.com
} 


$$
a(x)=\left\{\begin{array}{lll}
1 & \text { si } & x \in \omega, \\
0 & \text { si } & x \in(0,1)-\omega
\end{array}, \omega=(a, b) \subset(0,1)\right.
$$

Aquí ' denota diferenciación parcial con respecto al tiempo.

Observamos que $y^{0} \in H_{0}^{1}(0, \dot{1}), y^{1} \in L^{2}(0,1)$, entonces (1.1) tiene solución débil.

La energía

$$
E(t)=\frac{1}{2} \int_{0}^{1}\left\{\left|y^{\prime}(x, t)\right|^{2}+\left|y_{x}(x, t)\right|^{2}\right\} d x, \forall t \geq 0
$$

de la solución de la ecuación de onda amortiguada (1.1) tiene la siguiente ley de disipación:

$$
\begin{aligned}
\frac{d E(t)}{d t} & =-\int_{0}^{1} a(x)\left|\frac{\partial y}{\partial t}(x, t)\right|^{2} d x \\
& =-\int_{\omega}\left|\frac{\partial y}{\partial t}(x, t)\right|^{2} d x
\end{aligned}
$$

Observamos que el decaimiento de la energía depende del intervalo $\omega$.

Está probado en [1], [4] y [6] para función $a(x)$ definida en (1.4) la energía de la solución de (1.1) satisface para algún $t_{0}>0$ la desigualdad:

$$
E(t) \leq\left[\exp \left(1-\frac{t}{t_{0}}\right)\right] E(0), \forall t \geq t_{0}
$$

esta propiedad de decaimiento exponencial de la solución de (1.1) es equivalente a la Desigualdad de Observabilidad (O.I) para la solución del sistema no amortiguado con $a \equiv 0$. (ver [1]) Es decir, es equivalente a la existencia de un tiempo positivo $T$ y una constante positiva $C$ tal que:

$$
E(0) \leq C \int_{0}^{T} \int_{0}^{1} a(x)\left|y_{t}(x, t)\right|^{2} d x d t
$$

para cada solución $y(x, t)$ del sistema conservativo siguiente

$$
\left\{\begin{array}{lll}
y^{\prime \prime}-y_{x x}=0 & \text { en } & (0,1) \times(0, \infty) \\
y(0, t)=y(1, t)=0 & \text { en } & (0, \infty) \\
y(x, 0)=y^{0}(x) & \text { en } & (0,1) \\
y^{\prime}(x, 0)=y^{1}(x) & \text { en } & (0,1)
\end{array}\right.
$$

Analizaremos si con el modelo de aproximación numérica que consiste en el espacio de semidiscretización en diferencias finitas para el problema (1.1) se tiene la misma razón de decaimiento y si esta es uniforme con respecto al tamaño de paso de la semidiscretización.

Sea $N$ un número entero no negativo. Sea $h=\frac{1}{N+1}$ y consideremos la partición de $(0,1)$ dado por

$$
0=x_{0}<x_{1}<x_{2}<\cdots<x_{N}<x_{N+1}=1
$$

donde $x_{j}=j h, j=1,2, \ldots, N+1$.

El espacio de semidiscretización en diferencias finitas del sistema (1.1) es como sigue:

$$
\left\{\begin{array}{rll}
y_{j}^{\prime \prime}-\frac{y_{j+1}-2 y_{j}+y_{j-1}}{h^{2}}+a_{j} y_{j}^{\prime}=0 & , \quad t \in(0, \infty), j=1,2, \ldots, N \\
y_{0}(t)=y_{N+1}(t)=0 & , \quad t \in(0, \infty) \\
y_{j}(0)=y_{j}^{0}, y_{j}^{\prime}(0)=y_{j}^{1} & , j=1,2, \ldots, N
\end{array}\right.
$$

donde $a_{j}, y_{j}^{0}, y_{j}^{1}, j=0.1,2, \ldots, N+1$ son aproximaciones de las funciones $a, y^{0}, y^{1}$ respectivamente.

La energía del sistema (1.10) es dado por

$$
E_{h}(t)=\frac{h}{2} \sum_{j=0}^{N}\left\{\left(y_{j}^{\prime}(t)\right)^{2}+\left(\frac{y_{j+1}-y_{j}}{h}\right)^{2}\right\}
$$


Su derivada es dado por

$$
E_{h}^{\prime}(t)=-h \sum_{j=1}^{N} a_{j}\left(y_{j}^{\prime}(t)\right)^{2}
$$

Entonces $E_{h}(t)$ es una función no creciente del tiempo $t$. Esperamos determinar si la energía $E_{h}$ decae exponencialmente y uniformemente a cero con respecto a $h$ cuando $t \rightarrow \infty$

Consideramos el sistema conservativo asociado con (1.10)

$$
\begin{cases}u_{j}^{\prime \prime}-\frac{u_{j+1}-2 u_{j}+u_{j-1}}{h^{2}}=0 & \text { en }(0, \infty), j=1,2, \ldots, N \\ u_{0}(t)=u_{N+1}(t)=0 & \text { en } \quad(0, \infty) \\ u_{j}(0)=u_{j}^{0}, u_{j}^{\prime}(0)=u_{j}^{1} & ; \quad j=1,2, \ldots, N\end{cases}
$$

Para probar el decaimiento exponencial de soluciones del sistema semidiscreto amortiguado (1.10) es necesario y suficiente probar la existencia de un tiempo $T>0$ y una constante $C>0$ tal que la siguiente desigualdad de observabilidad $\left(O \cdot I_{h}\right)$ se cumple:

$$
E_{h}(0) \leq C \int_{0}^{T}\left[h \sum_{j=1}^{N} a_{j}\left|u_{j}^{\prime}\right|^{2}\right] d t
$$

para cada solución $u$ de (1.13) .

Además, la razón de decaimiento exponencial es uniforme con respecto al tamaño de paso $h$ si y sólo si el tiempo $T$ y la constante $C$ en esta desigualdad son independientes de $h$.

Sin embargo fue probado en [2] y [3] que este tipo de desigualdad no es uniforme en $h$ cuando $h \rightarrow 0$, en particular, si se hace la observación sobre la frontera del intervalo $(0,1)$, y en el cáso del extremo $x=1$, reemplazando el lado derecho de la desigualdad (1.14) por $\int_{0}^{T}\left|\frac{u_{N}}{h}\right|^{2} d t$. Por eso, las soluciones de (1.10) no decaen exponencialmente a cero cuando $t \rightarrow \infty$ con una razón de decaimiento uniforme.

Por eso, para obtener decaimiento uniforme, sumamos en el sistema (1.10) un adecuado término de viscosidad numérica como lo que sigue:

$$
\left\{\begin{array}{rll}
y_{j}^{\prime \prime}-\frac{y_{j+1}-2 y_{j}+y_{j-1}}{h^{2}}-h^{2}\left(\frac{y_{j+1}^{\prime}-2 y_{j}^{\prime}+y_{j-1}^{\prime}}{h^{2}}\right)+a_{j} y_{j}^{\prime}=0 & \text { en } & (0, \infty) \\
y_{0}(t)=y_{N+1}(t)=0 & \text { en } & (0, \infty) \\
y_{j}(0)=y_{j}^{0}, y_{j}^{\prime}(0)=y_{j}^{1} & ; \quad j=1,2, \ldots, N
\end{array}\right.
$$

La energía de este nuevo sistema es $E_{h}$ y su derivada es

$$
E_{h}^{\prime}(t)=-h^{3} \sum_{j=0}^{N}\left\{\left(\frac{y_{j+1}^{\prime}-y_{j}^{\prime}}{h}\right)^{2}-h \sum_{j=1}^{N} a_{j}\left(u_{j}^{\prime}(t)\right)^{2}\right\}
$$

Resulta que este sistema es un buen esquema de aproximación para (1.1): garantiza la convergencia de soluciones cuando $h \rightarrow 0$, y también, da una razón de decaimiento uniforme de soluciones cuando $t \rightarrow \infty$.

\section{Resultados Principales:}

Teorema 2.1 Sea $a(x)$ la función dada en (1.4) y consideremos el sistema (1.15) y la energía $E_{h}$ respectiva. Existen constantes positivas $M$ y $t_{1}$ independientes de $h$ tal que $E_{h}$ satisface

$$
E_{h} \leq M\left(\exp -t_{1} t\right) E_{h}(0), \forall t \geq 0
$$

para cada solución del sistema (1.15) y cada $h$ tal que $0<h<1$.

\section{Observaciones:}

1. En la desigualdad (2.1) tenemos la razón de decaimiento exponencial uniforme, dado que

$$
\frac{E_{h}(t)}{E_{h}(0)} \leq M\left(\exp -t_{1} t\right)
$$

$M\left(\exp -t_{1} t\right)$ tiende a cero cuando $t \rightarrow \infty$ independientemente de $h$. 
2. En la demostración del Teorema 2.1, primero se obtiene una adecuada desigualdad de observabilidad uniforme con respecto a $h$, la cual implicará la desigualdad (2.1). Ver [5].

Antes de enunciar el segundo resultado necesitamos las notaciones adicionales siguientes.

Sean

análogamente

$$
a_{h}=\left(a_{j}\right)_{j}=\left(a_{1}, a_{2}, \ldots, a_{N+1}\right) \quad y_{h}=\left(y_{j}\right)_{j}=\left(y_{1}, y_{2}, \ldots, y_{N+1}\right) \text { en } \mathbb{R}^{n+1}
$$

$$
y_{h}^{0}=\left(y_{j}^{0}\right)_{j}, y_{h}^{1}=\left(y_{j}^{1}\right)_{j} \text { en } \mathbb{R}^{n+1} .
$$

Sea $v_{h}=\left(v_{0}, v_{1}, \ldots, v_{N+1}\right) \in \mathbb{R}^{n+2}$, definimos los operadores:

$$
\begin{gathered}
p_{h} v_{h}=\left\{\begin{array}{l}
\text { la función continua lineal en cada intervalo }[j h,(j+1) h] \text { tal que: } \\
\left(p_{h} v_{h}\right)(j h)=v_{j}, j=0,1,2, \ldots, N+1
\end{array}\right. \\
q_{h} v_{h}=\left\{\begin{array}{l}
\text { la función escalonada definida en cada intervalo }\left(\left(j-\frac{1}{2}\right) h,\left(j+\frac{1}{2}\right) h\right) \cap(0,1), \\
\text { por }\left(q_{h} v_{h}\right)(x)=v_{j}, j=0,1,2, \ldots, N+1
\end{array}\right.
\end{gathered}
$$

Entonces

$$
\begin{gathered}
\int_{0}^{1} \frac{d}{d x}\left(p_{h} v_{h}\right) \frac{d}{d x}\left(p_{h} w_{h}\right) d x=h \sum_{j=0}^{N}\left(\frac{v_{j+1}-v_{j}}{h}\right)\left(\frac{w_{j+1}-w_{j}}{h}\right) \\
\int_{0}^{1}\left(q_{h} v_{h}\right)\left(q_{h} w_{h}\right) d x=h \sum_{j=0}^{N}\left(v_{j}\right)\left(w_{j}\right)
\end{gathered}
$$

Teorema 2.2 Sea $y_{h}$ que denota la solución de (1.12). Consideremos las funciones $y^{0}, y^{1}, a$ definidas en (1.2); (1.3), (1.4). Para $h$ tal que $0<h<1$, asumimos que $a_{h}, y_{h}^{0}$ y $y_{h}^{1}$ son tales que existe una constante $C$ no negativa independiente de $h$ con

$$
\begin{gathered}
E_{h}(0) \leq C, q_{h} a_{h} \rightarrow a \text { débil }^{*} \text { en } L^{\infty}(0,1) \\
p_{h} y_{h}^{0} \rightarrow y^{0} \text { débil en } H_{0}^{1}(0,1), \\
q_{h} y_{h}^{1} \rightarrow y^{1} \text { débil en } L^{2}(0,1),
\end{gathered}
$$

cuando $h$ tiende a cero.

Entonces existe $y \in L^{\infty}\left(0, \infty ; H_{0}^{1}(0,1)\right)$ tal que

$$
\begin{aligned}
& p_{h} y_{h} \rightarrow y \text { débil }^{*} \text { en } L^{\infty}\left(0, \infty ; H_{0}^{1}(0,1)\right) \\
& q_{h} y_{h}^{\prime} \rightarrow y^{\prime} \text { débil }^{*} \text { en } L^{\infty}\left(0, \infty: L^{2}(0,1)\right)
\end{aligned}
$$

e $y$ es la solución del sistema (1.1).

\section{Observaciones}

1. De la hipótesis (2.6) tenemos $q_{h} a_{h} \rightarrow a$ débil* en $L^{\infty}(0,1)$. Como $L^{\infty}(0.1)=\left(L^{1}(0,1)\right)^{\prime}$ entonces

$$
\int_{0}^{1} q_{h} a_{h} f d x \rightarrow \int_{0}^{1} a f d x, \forall f \in L^{1}(0,1) \Leftrightarrow \int_{\omega} q_{h} a_{h} d x \rightarrow \int_{\omega} \text { afd } d x, \forall f \in L^{1}(0,1)
$$

2. De la hipótesis (2.7): como $H_{0}^{1}(0,1)$ es un espacio de Hilbert tenemos:

$$
\left\langle p_{h} y_{h}^{0}, f\right\rangle \rightarrow\left\langle y^{0}, f\right\rangle, \forall f \in H_{0}^{1}(0,1) ; \text { donde }\langle\cdot, \cdot\rangle \text { denota el producto interior de } H_{0}^{1}(0,1)
$$

3. De la hipótesis $(2.8)$ : como $L^{2}(0,1)$ es un espacio de Hilbert

$$
\left\langle q_{h} y_{h}^{1}, f\right\rangle \rightarrow\left\langle y^{1}, f\right\rangle, \forall f \in L^{2}(0,1): \text { donde }\langle\cdot, \cdot\rangle \text { denota el producto interior de } L^{2}(0,1)
$$

es decir

$$
\int_{0}^{1} q_{h} y_{h}^{1} f d x \rightarrow \int_{0}^{1} y^{1} f d x ; \forall f \in L^{2}(0,1)
$$




\section{Demostración del Teorema 2.2}

Tenemos que

$$
E_{h}(t)=\frac{h}{2} \sum_{j=0}^{N}\left\{\left(\frac{y_{j}^{\prime}(t)}{h}\right)^{2}-\left(\frac{y_{j+1}(t)-y_{j}(t)}{h}\right)^{2}\right\},
$$

entonces

$$
E_{h}(t)=\frac{1}{2}\left(\left\|p_{h} y_{h}(t)\right\|_{H_{0}^{1}(0,1)}^{2}+\left\|q_{h} y_{h}^{\prime}(t)\right\|_{L^{2}(0,1)}^{2}\right)
$$

Como $E_{h}(t)$ es decreciente, existe $M>0$ tal que $E_{h}(t) \leq M, \forall t \in(0, \infty)$; entonces

$$
\left\|p_{h} y_{h}(t)\right\|_{H_{0}^{1}(0,1)}^{2} \leq E_{h}(t) \leq M,
$$

de donde $\left\|p_{h} y_{h}\right\|_{L^{\infty}\left(0, \infty ; H_{0}^{1}(0,1)\right)}^{2} \leq \sqrt{M}$. Es decir $p_{h} y_{h}$ es acotado en $L^{\infty}\left(0, \infty ; H_{0}^{1}(0,1)\right)$; y extrayendo una subsucesión que volvemos a denotar con $p_{h} y_{h}$, se tiene que existe $y \in L^{\infty}\left(0, \infty ; H_{0}^{1}(0,1)\right)$ tal que

$$
p_{h} y_{h} \rightarrow y \text { débil }^{*} \text { en } L^{\infty}\left(0, \infty ; H_{0}^{1}(0,1)\right)
$$

Análogamente $q_{h} y_{h}$ es acotado en $L^{\infty}\left(0, \infty ; L^{2}(0,1)\right)$ y extrayendo una subsucesión que volvemos denotar con $q_{h} y_{h}$ se tiene:

$$
q_{h} y_{h} \rightarrow y \text { débil }^{*} \text { en } L^{\infty}\left(0, \infty ; L^{2}(0,1)\right)
$$

Adicionalmente se tiene los resultados:

$$
\begin{gathered}
p_{h} y_{h}^{\prime} \rightarrow y^{\prime} \text { débil }^{*} \text { en } L^{\infty}\left(0, \infty ; L^{2}(0,1)\right) \\
p_{h} y_{h} \rightarrow y \text { fuertemente en } L_{\mathrm{loc}}^{2}\left(0, \infty ; L^{2}(0,1)\right) \\
q_{h} y_{h}^{\prime} \rightarrow y^{\prime} \text { débil }^{*} \text { en } L^{\curlyvee}\left(0, \infty ; L^{2}(0,1)\right) \\
h p_{h} y_{h}^{\prime} \rightarrow 0 \text { débil }^{*} \text { en } L^{2}\left(0, \infty ; H_{0}^{1}(0,1)\right)
\end{gathered}
$$

Se tiene que probar que el limite y es la solución de (1.1): sea $w \in \mathcal{D}((0,1) \times(0, \infty))$ y sea $w_{h}=\left(w_{j}\right)_{j}$ donde $w_{j}=w(j h, t)$. Multiplicando la primera ecuación de (1.15) por $w_{j}$, integrando por partes sobre $(0, \infty)$, tomando la suma sobre $j$ y nsando definiciones de $p_{h}$ y $q_{h}$ tenemos

$$
\begin{aligned}
\int_{0}^{\infty} \int_{0}^{1}\left(q_{h} y_{h}\right)\left(q_{h} w_{h}^{\prime \prime}\right) d x d t & +\int_{0}^{\infty} \int_{0}^{1}\left(p_{h} y_{h}\right)_{x}\left(p_{h} w_{h}\right)_{x} d x d t \\
& +h^{2} \int_{0}^{\infty} \int_{0}^{1}\left(p_{h} y_{h}^{\prime}\right)_{x}\left(p_{h} w_{h}\right)_{x} d x d t-\int_{0}^{\infty} \int_{0}^{1}\left(q_{h} y_{h}\right)\left(q_{h} w_{h}^{\prime}\right)\left(q_{h} a_{h}\right) d x d t=0
\end{aligned}
$$

Por otra parte: para cada $w \in \mathcal{D}((0,1) \times(0, \infty))$

$$
\left\{\begin{array}{l}
p_{h} y_{h} \rightarrow \text { fuertemente en } L^{2}\left(0, \infty ; H_{0}^{1}(0,1)\right) \\
p_{h} w_{h} \rightarrow \text { fuertemente en } L^{2}\left(0, \infty ; L^{2}(0.1)\right)
\end{array}\right.
$$

De (2.20), (2.13) y (2.18) podemos pasar al limite en todos los términos en (2.19) obteniendo

$$
\int_{0}^{\infty} \int_{0}^{1} y v^{\prime \prime} d x d t+\int_{0}^{\infty} \int_{0}^{1} y_{x} w_{x} d x d t-\int_{0}^{\infty} \int_{0}^{1} y w^{\prime} a d x d t=0
$$

entonces

$$
\int_{0}^{\infty} \int_{0}^{1}\left(y^{\prime \prime}-y_{x x}+a y^{\prime}\right) w d x d t=0 . \forall w \in \mathcal{D}((0,1) \times(0, \infty))
$$


Para probar que $y(0)=y^{0}, y^{\prime}(0)=y^{1}$, consideramos $v \in \mathcal{D}(0,1)$ y $\ell \in \mathcal{D}(0, \infty)$, y sea $v_{h}=\left(v_{j}\right)_{j}$ donde $v_{j}=v(j h)$. Multiplicando la primera ecuación de (1.15) por $v_{j} \ell$, integrando por partes sobre $(0, \infty)$, tomando la suma sobre $j, \mathrm{y}$ usando las definiciones de $p_{h}$ y $q_{h}$, se obtiene:

$$
\begin{aligned}
-\ell(0) \int_{0}^{1}\left(q_{h} y_{h}^{1}\right)\left(q_{h} v_{h}\right) d x & +\ell^{\prime}(0) \int_{0}^{1}\left(q_{h} y_{0}^{1}\right)\left(q_{h} v_{h}\right) d x \\
-\ell(0) \int_{0}^{1}\left(q_{h} y_{h}^{0}\right)\left(q_{h} v_{h}\right)\left(q_{h} a_{h}\right) d x+\int_{0}^{\infty} \int_{0}^{1}\left(q_{h} y_{h}\right)\left(q_{h} v_{h}\right) \ell^{\prime \prime} d x d t & \\
+\int_{0}^{\infty} \int_{0}^{1}\left(p_{h} y_{h}\right)_{x}\left(p_{h} v_{h}\right)_{x} \ell d x d t & +h^{2} \int_{0}^{\infty} \int_{0}^{1}\left(p_{h} y_{h}^{\prime}\right)_{x}\left(p_{h} v_{h}\right)_{x} \ell d x d t \\
& -\int_{0}^{\infty} \int_{0}^{1}\left(q_{h} y_{h}\right)\left(q_{h} v_{h}\right)\left(q_{h} a_{h}\right) \ell^{\prime} d x d t=C
\end{aligned}
$$

Pasando al limite como $h \rightarrow 0$ en (2.22), obtenemos

$$
\begin{aligned}
-\ell(0) \int_{0}^{1} y^{1} v d x+\ell^{\prime}(0) \int_{0}^{1} y^{0} v d x- & \ell(0) \int_{0}^{1} y^{0} v a d x \\
& +\int_{0}^{\infty} \int_{0}^{1} y v \ell^{\prime \prime} d x d t+\int_{0}^{\infty} \int_{0}^{1} y_{x} v_{x} \ell d x d t-\int_{0}^{\infty} \int_{0}^{1} y v a \ell^{\prime} d x d t=0
\end{aligned}
$$

desde el cual deducimos $y(0)=y^{0}, y^{\prime}(0)=y^{1}$.

\section{Conclusiones:}

(i) Del sistema (1.15) obtenemos una razón de decaimiento para la energía similar a la razón de decaimiento en (1.7): el cual es uniforme con respecto al tamaño de paso $h$ en la semidiscretización.

(ii) Del sistema (1.15), la convergencia de sus soluciones es a las soluciones de la ecuación de onda (1.1) cuando $h \rightarrow 0$, en el espacio de Sobolev $L^{2}\left(0, \infty ; H_{0}^{1}(0,1)\right)$. 


\section{Bibliografía}

[1] Haraux, A. (1989) Une remarque sur la stabilisation de certains systemes du deuxieme ordre eñ Temps, Portugal Math. 46, 245 -258.

[2] Infante, J.A., Zuazua, E. (1997) Boundary observability for the space-discretizations of the 1-D wave equation. Acad. Paris, Serie I, 326, 713 -718.

[3] Infante, J.A., ZuAzuA, E. (1999) Boundary observability for the space semi-discretizations of the 1-D wave equation. Math. Model. Num. 33, 407-438.

[4] Tcheugoué Tebou, L. R, Estimation énergie power Lequation des ondes avec un amortissement nonlinéaire Localisé, C.R. Acad. Paris, serie J 325. 1175 -1179.

[5] Tcheugoué Tébou, L. R., Zuazua, E. (2003) Uniform exponential long time decay for the space semidiscretization of a locally damped wave equation vía and artificial numerical viscosity, Numer Math 95, 563598. Published online February 18.

[6] Zuazua, E. (1990) Exponential decay for the semilinear wave equation with locally distributed damping, Commun, in P.D.E. (15), 205-235. 